\title{
Modelo matemático e meta-heurística Simulated Annealing para o problema de alocação de berços com múltiplas cargas
}

\author{
Rafael Silva Banos ${ }^{1}$, Rodrigo de Alvarenga Rosa², Geraldo Regis Mauri ${ }^{3}$ e Glaydston Mattos Ribeiro ${ }^{4}$
}

\begin{abstract}
Resumo: Ocorre no Brasil um forte crescimento do comércio exterior na área de granéis, como minério de ferro e agrícolas que são exportados pelos portos. Assim, metodologias que auxiliem o planejamento da operação dos portos são importantes. Este artigo propõe um modelo matemático aplicado a um porto de granel que difere dos demais por três itens: 1) berços operam mais de um tipo de carga e com taxas de operação diferentes para cada um, 2) certas cargas não são operadas em todos os berços, 3) o tempo de operação é dependente do berço e da carga. O modelo proposto elabora a sequência de atendimento dos navios em cada berço e foi implementado no CPLEX 12.6. Como soluções ótimas são difíceis de serem alcançadas, é proposta também uma meta-heurística Simulated Annealing (SA). Para avaliação do modelo e do SA, foram realizados testes com dados reais do Complexo Portuário de Tubarão.

Palavras-chave: Problema de Alocação de Berços com Múltiplas Cargas, Simulated Annealing, Operação Portuária.
\end{abstract}

\begin{abstract}
Brazil has been facing a strong growth of foreign trade in bulk area, such as iron ore and agricultural which are exported through the ports. Thus, methodologies that assist the planning of the operation of the ports are important. This paper proposes a mathematical model applied to a bulk port that differs from the others in three points: 1) berths operate more than one type of cargo with different operating rates for each, 2) certain cargos cannot be operated at all berths, 3) the operating time is dependent of the berth and the cargo. The proposed model elaborates the ship service sequence in each berth and it was implemented in CPLEX 12.6. Since optimal solutions are difficult to reach, it is also proposed a metaheuristic Simulated Annealing (SA). To evaluate the model and the SA, it was carried out tests with real data of the Port of Tubarão Complex.
\end{abstract}

Keywords: Berth Allocation Problem with Multiple Cargos, Simulated Annealing, Port Operation.

\section{INTRODUÇÃO}

A principal função do transporte marítimo de cargas é promover a ligação entre regiões produtoras e consumidoras de insumos e produtos. Isso inclui transporte de matérias-primas, como, por exemplo, minério de ferro, óleo bruto, carvão, grãos e insumos para a produção agrícola, bem como produtos manufaturados. No Brasil, o setor portuário movimenta anualmente cerca de 900 milhões de toneladas de carga e responde por $95 \%$ das exportações do país (ANTAQ, 2013).

A carga a granel é uma carga caracterizada por não haver como identificar cada unidade de carga e, assim sendo, sua movimentação é registrada por volume ou peso. Essas cargas são transportadas soltas, sem embalagem e geralmente em fluxo contínuo, ou seja, por meio de transportadores de correia ou dutos. Dentre as cargas a granel, destacam-se o mineiro de ferro, as cargas agrí-

\footnotetext{
Rafael Silva Banos, Departamento de Engenharia de Produção, Universidade Federal do Espírito Santo, Vitória, ES, Brasil (rafaelsilvabanos@hotmail.com).

${ }^{2}$ Rodrigo de Alvarenga Rosa, Programa de Pós-Graduação em Engenharia Civil, Universidade Federal do Espírito Santo, Vitória, ES, Brasil (rodrigo.a.rosa@ufes.br)

${ }^{3}$ Geraldo Regis Mauri, Programa de Pós-Graduação em Informática, Universidade Federal do Espírito Santo, Alegre, ES, Brasil (geraldo.mauri@ufes.br).
}

${ }^{4}$ Glaydston Mattos Ribeiro, Programa de Pós-Graduação em Engenharia de Transportes, Universidade Federal do Rio de Janeiro, Rio de Janeiro, RJ, Brasil (glaydston@pet.coppe.ufrj.br).

Manuscrito recebido em 18/08/2015 e aprovado para publicação em $15 / 01 / 2016$

Este artigo é parte de TRANSPORTES v. 24, n. 1, 2016 ISSN: 2237-1346 (online). DOI:10.14295/transportes.v24i1.980 colas (soja, farelo de soja, milho, trigo, fertilizante), o carvão mineral dentre outras.

Assim, os portos graneleiros são usualmente projetados e construídos de forma específica para uma ou mais cargas que se pretende operar com taxas operacionais, toneladas/hora ou litros/hora, específicas para cada volume de carga operada. Dessa forma, eles são projetados para certa demanda de projeto e com a tecnologia de equipamentos da época do projeto. Porém, após o início da operação, alguns desses portos realizam expansões construindo novos berços que incorporam novos equipamentos de carregamento e descarga o que leva aos berços mais modernos terem, muitas das vezes, taxas operacionais maiores permitindo o carregamento e/ou o descarregamento dos navios de forma mais rápida.

Pelo exposto anteriormente, pode-se perceber que um porto de granel usualmente tem berços que atendem a uma ou mais cargas, dependendo da compatibilidade entre elas, e que podem ter taxas operacionais diferentes para diferentes cargas. Também pode ocorrer que para uma mesma carga, a taxa operacional de um berço seja maior ou menor que nos outros berços. Assim, os planejadores do porto devem, então, planejar a sequência de atracação dos navios no porto de tal forma que os navios atraquem em berços que possam operar sua carga. Como mencionado, sabe-se que o tempo de operação do navio é dependente da carga transportada pelo navio e do berço que o navio irá atracar e, assim, o planejador do porto deve definir para cada navio, o berço que tenha a melhor taxa operacional de tal forma que se obtenha a redução total do tempo de espera para atracar de todos os navios que vão operar no porto.

Para elaborar o planejamento da sequência de atracação dos navios em cada berço do porto, foi proposto na literatura o Problema de Alocação de Berços (PAB) que se 
refere basicamente ao problema de planejar a sequência de atendimento de um conjunto de navios em cada berço dentre aqueles disponíveis no porto em certo horizonte de tempo de planejamento atendendo às restrições operacionais e comerciais do porto. O objetivo mais usual é minimizar o tempo de permanência dos navios no porto, ou seja, reduzir o tempo de espera para atracar mais o tempo para operar (Bierwirth e Meisel, 2010). Em função dos altos custos operacionais dos portos e dos navios, a comunidade científica tem dedicado especial atenção ao PAB e diversos artigos já foram publicados trazendo revisões sobre o tema. Dentre estas revisões, citam-se Meersmans e Dekker (2001), Vis e de Koster (2003), Steenken et al. (2004), Vacca et al. (2007), Stahlbock e Voss (2008), Bierwirth e Meisel (2010) e Rashidi e Tsang (2013).

Visando contribuir com a pesquisa acerca do PAB, estudando um problema característico dos portos graneleiros, este artigo apresenta um modelo matemático e uma meta-heurística Simulated Annealing (SA) para tratar o PAB em portos que operam navios com diferentes cargas e que as taxas operacionais dependem da carga e do berço que o navio irá operar. Ressaltando que cada berço não opera todas as cargas, ou seja, nem todo navio pode operar em todos os berços. Desta forma, o PAB tratado neste artigo não trata o tempo de operação como determinístico e conhecido $a$ priori, mas sim, considera o tempo de operação dependente do berço que o navio irá atracar e da carga que ele transporta. Esse problema foi originalmente tratado por Rosa et. al. (2013), que denominaram o problema como Problema de Alocação de Berços com Múltiplas Cargas (PAB-MC) e, neste artigo, é proposta uma nova formulação matemática para o problema baseada no Problema de Roteamento de Veículos com Múltiplos Depósitos e com Janela de Tempo (PRV-MD-JT) proposto por Cordeau et al. (2005). A diferença entre o modelo matemático proposto neste artigo e o modelo proposto por Cordeau et al. (2005) é que o modelo de Cordeau et al. (2005) tem por premissa que os tempos de operação dos navios nos berços são conhecidos a priori e no modelo matemático proposto neste artigo, o tempo de operação é calculado e depende da relação entre a carga transportada pelo navio e o berço que ele irá atracar. Portanto, o tempo de operação não é conhecido a priori. Esta situação torna o modelo matemático não linear e, por isso, é apresentada, também, uma linearização do modelo.

Sabendo-se que o Problema de Roteamento de Veículos (PRV) é considerado NP-Hard e, por conseguinte, o PRV-MD-JT também é NP-Hard, infere-se que o modelo proposto neste artigo também seja NP-Hard. Assim, problemas NP-Hard são problemas difíceis de encontrar soluções ótimas devido à sua complexidade combinatória, sobretudo, para instâncias maiores e, então, neste artigo é apresentada uma meta-heurística Simulated Annealing para o PAB-MC.

Para avaliar o modelo proposto, o mesmo foi aplicado ao Complexo Portuário de Tubarão que é um dos maiores complexos portuários brasileiros e o maior porto de movimentação de minério de ferro do mundo. O complexo portuário é composto pelo Porto de Tubarão, Terminal de Praia Mole e Terminal de Produtos Diversos. Eles atendem à área de mineração, minério de ferro e carvão mineral, e a área de granéis agrícolas, soja, farelo de soja, milho e fertilizantes. Na Figura 1 tem-se uma vista aérea dos berços do Complexo Portuário de Tubarão que possui sete berços de atraca- ção. O planejamento de atracação dos sete berços é realizado de forma integrada por um único Centro de Controle Operacional do complexo portuário. O Píer I Sul e o Pier I Norte realizam carregamento de minério de ferro e pelota a uma taxa operacional de carregamento de $13.000,0$ ton/hora admitindo navios, respectivamente, com calado até $16,0 \mathrm{~m}$ e $17,0 \mathrm{~m}$ e comprimento máximo de $285,0 \mathrm{~m}$ e $301,0 \mathrm{~m}$. O Pier II realiza carregamento de minério de ferro e pelota a uma taxa operacional de carregamento de 16.000,0 ton/hora admitindo navios com calado até $26,0 \mathrm{~m}$ e comprimento máximo de 400,0m. O Píer III é especializado em carregamento de granéis agrícolas como soja, farelo de soja e milho e opera a uma taxa de 3.000 ton/hora para soja e milho e 2.500 ton/hora para farelo de soja admitindo navios com até $15,0 \mathrm{~m}$ de calado e comprimento máximo de 280,0m. O Píer IV opera o descarregamento de fertilizantes a uma taxa de $1.500,0$ ton/hora admitindo navios com até $12,0 \mathrm{~m}$ de calado e comprimento máximo de $245,0 \mathrm{~m}$. O Pier de Praia Mole opera o descarregamento do carvão mineral e é dividido em dois berços que admitem navios com até $16,0 \mathrm{~m}$ de calado e 300,0m de comprimento máximo (SINDAMARES, 2013).

$\mathrm{O}$ artigo está organizado em seis seções. Na Seção 2 é apresentado o Problema de Alocação de Berços bem como uma revisão bibliográfica. As Seções 3 e 4 descrevem o modelo matemático e a meta-heurística propostos, respectivamente. Na Seção 5, são apresentados os resultados e é feita a análise das soluções e discussão dos pontos pertinentes. Por fim, na Seção 6 é realizada a conclusão do trabalho.

\section{PROBLEMA DE ALOCAÇÃO DE BERÇOS (PAB)}

O PAB é definido como sendo o problema de atender a um conjunto de navios num conjunto de berços de um porto em um horizonte de tempo de planejamento. Ao fim, tem-se a sequência de atendimento aos navios. Assim, as principais decisões a serem tomadas neste processo envolvem a escolha de onde e quando os navios deverão atracar (CORDEAU et al., 2005). O objetivo mais usual é minimizar a soma do tempo de espera para atracar mais o tempo de operação dos navios, isto é, o tempo de permanência no porto (BIERWIRTH e MEISEL, 2010).

Os modelos existentes para o PAB na literatura podem ser classificados tanto por restrições espaciais como por restrições temporais. Quanto aos atributos espaciais, relativos ao layout do cais, usualmente adotam-se as restrições de calado, comprimento e boca dos navios. As restrições espaciais também podem restringir as posições viáveis de atracação de navios. Caso ocorra um pré-particionamento do cais em berços, então o problema é denominado PAB discreto (PABD). Em contrapartida, no $\mathrm{PAB}$ Contínuo (PABC) não há divisão do cais, ou seja, os navios podem atracar em posições arbitrárias dentro dos limites do cais. Rodrigues et al. (2015) analisaram o PABC onde não há divisão do cais, porém há restrições de atracações pela descontinuidade do cais.

Dentre os atributos temporais, podem ser citados: tempo de chegada de navio, tempo de atracação, tempo de espera na fila de navios, dentre outros. Restrições temporais ocorrem principalmente em relação aos tempos de atracação e de desatracação dos navios. De acordo com Imai et al. (2001), as restrições temporais podem ser classificadas em chegada estática e chegada dinâmica. A chegada dinâmica 


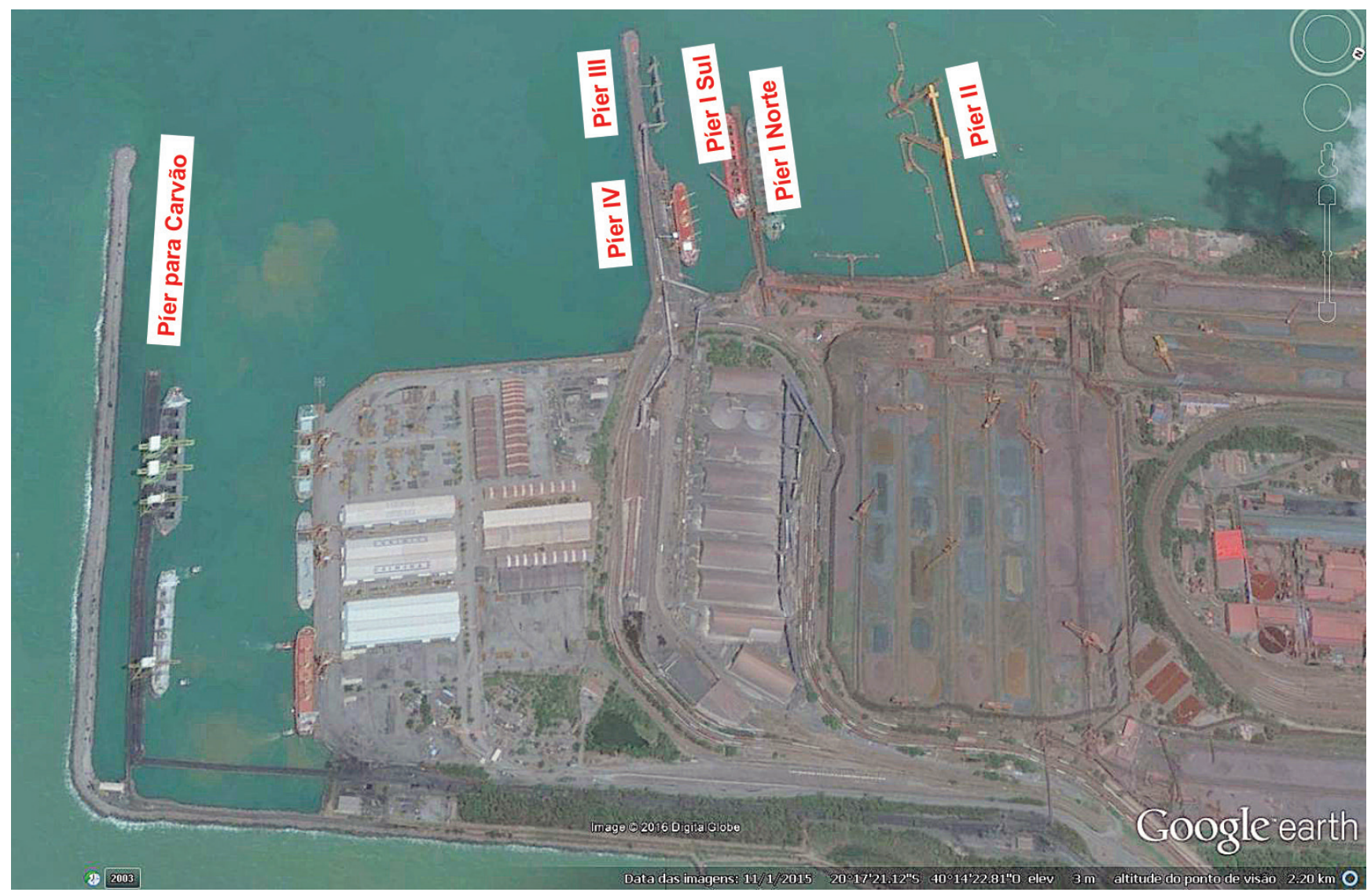

Figura 1: Vista geral do Porto de Tubarão e identificação dos berços

representa o cenário da maioria dos portos, no qual os navios têm tempos definidos de chegada e não têm permissão para atracar antes deste tempo. No caso de chegada estática, não há considerações quanto aos tempos de chegada dos navios. Isto ocorre porque se considera que todos os navios estão esperando na área de fundeio do porto, na espera de liberação para atracação, e podem atracar imediatamente (BIERWIRTH e MEISEL, 2010).

Os tempos de chegada dos navios podem ser considerados como determinístico ou estocástico. Nas chegadas determinísticas, os tempos de chegada são fixados como parâmetros do problema. Já nas chegadas estocásticas, consideram-se as incertezas no tempo de chegada dos navios decorrentes de problemas ocorridos em outros portos ou problemas de navegação. Neste caso, os tempos são estimados com base em dados históricos e curvas estatísticas de distribuição do tempo de chegada. Outra abordagem, na qual o tempo de operação depende do berço em que o navio irá atracar, também pode ser utilizada.

Diversas abordagens para resolução do PABD foram propostas, a seguir é apresentada uma revisão bibliográfica dos principais artigos sobre o PABD. Cordeau et al. (2005) modelaram o PABD como o PRV-MD-JT e propuseram uma meta-heurística Tabu Search para o problema. O PRV-MD-JT é também a base de Mauri et al. (2008a, 2008b, 2010), que propuseram a meta-heurística SA e o método de Geração de Colunas na resolução do PABD. Rodrigues et al. (2013) propuseram a aplicação do SA proposto por Mauri et al. (2008a) a um porto brasileiro. Hansen et al. (2008) propuseram uma meta-heurística Variable Neighborhood Search. Oliveira et al. (2012) propuseram uma meta-heurística Clustering Search. Barros et al. (2011) apresentaram um modelo para portos graneleiros considerando variação de maré e utilizaram a meta-heurística SA.

Imai et al. (1997), Imai et al. (2001) e Imai et al. (2008) e Theofanis et al. (2007) analisaram o PABD com chegada estática com a função objetivo que visa minimizar o tempo total de serviços dos navios e os desvios entre a sequência de chegada e a sequência de atracação dos navios. Basicamente, eles trataram o PAB como um problema de atribuição e sequenciamento de navios para berços tendo como objetivo minimizar o tempo de espera para atracação dos navios. Hansen e Oguz (2003) propuseram um modelo de Programação Linear Inteira Mista mais compacto para o mesmo problema. Imai et al. (2001), Monaco and Sammarra (2007) e Imai et al. (2003) estudaram o PABD com chegada dinâmica. Zhou and Kang (2008), Golias et al. (2006 e 2007) e Han et al. (2010) lidaram com o PABD com chegada dinâmica que considera a data de chegada e o tempo de carregamento com comportamento estocástico. Zhou et al. (2006) e Han et al. (2006) consideraram a chegada dos navios como estocástica e que existe uma restrição de tempo de espera na fila de navios. Ambos utilizaram Algoritmo Genético para resolver o problema. Vervloet e Rosa (2016) propuseram um PABD que leva em consideração as questões comerciais relativas a uma Charter Party. Teixeira e Cunha (2012) analisaram a distribuição de contêineres vazios e seu impacto no porto.

\section{MODELO MATEMÁTICO PROPOSTO}

O modelo matemático proposto neste artigo para resolver o Problema de Alocação de Berços com Múltiplas Cargas (PAB-MC) é baseado no modelo matemático pro- 


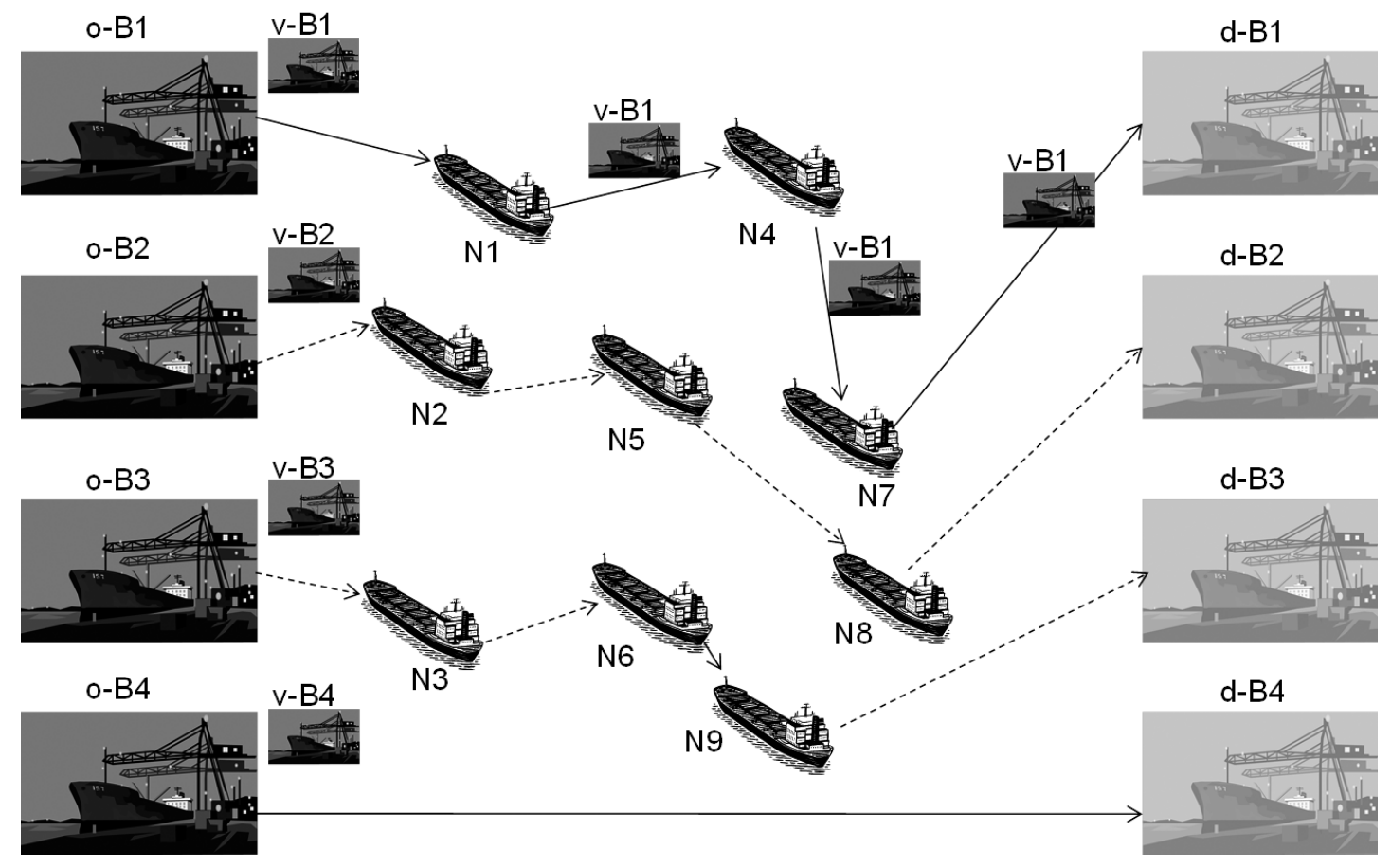

Figura 2: Desenho esquemático do modelo matemático PAB-MC

posto por Cordeau et al. (2005) que adaptou o PRV-MD-JT para resolver o PAB. A diferença entre o modelo matemático proposto neste artigo e o modelo proposto por Cordeau et al. (2005) é que eles definiram que os tempos de operação dos navios nos berços são conhecidos a priori e no modelo matemático proposto neste artigo, o tempo de operação depende da relação entre o berço que o navio irá atracar e da carga transportada, portanto, o tempo de operação não é conhecido a priori. Esta situação torna o modelo matemático não linear e, por isso, será apresentado a seguir o modelo matemático não linear e sua linearização.

O problema tratado neste artigo considera que os navios têm seu tempo de chegada ao porto conhecido. Portanto, eles não podem atracar nos berços antes dos prazos de chegada definidos. Os berços possuem restrições espaciais, ou seja, possuem profundidade e comprimento que limitam os navios a atracarem no mesmo. Somente é possível um navio atracar em um berço caso ele possua o comprimento e o calado menores que o comprimento e a profundidade do berço, respectivamente.

Para explicar o modelo matemático proposto, é apresentada a Figura 2 onde pode ser visto que cada berço é considerado um depósito e que cada berço possui um único veículo virtual. Cada navio é considerado um cliente a ser atendido por um único veículo, ou seja, é atendido por um único berço. Cada veículo de um berço faz uma rota que se inicia no depósito e termina em um depósito virtual que é o espelho deste ponto de origem. O tempo de viagem do veículo entre um navio 1 e um navio 2 é o tempo de operação do navio 1.

Assim, analisando na Figura 2 o berço 1, tem-se que o veículo v-B1 sai do depósito o-B1 e atende primeiro o navio $\mathrm{N} 1$, depois atende o navio $\mathrm{N} 4$, depois o navio $\mathrm{N} 7 \mathrm{e}$ finalmente segue para o depósito virtual d-B1, que é o espelho do depósito o-B1. O tempo de viagem entre o-B1 e N1 é zero e o tempo de viagem entre N1 e N4 é o tempo de ope- ração de N1 e, assim, respectivamente para todos os navios, inclusive de N7 para d-B1. Para o berço o-B4, nota-se que o veículo v-B4 sai do depósito e segue direto para d-B4, ou seja, nenhum navio atracou no berço 4 , pois o veículo v-B4 não atendeu a nenhum navio.

Com base nas explicações anteriores, passa-se a definir formalmente o modelo matemático proposto. Seja $V$ um conjunto de navios planejados para chegar ao porto, $B$ um conjunto de berços e $C$ um conjunto de cargas que podem ser operadas pelo porto. Cada navio $i \in V$ possui os seguintes parâmetros: $a_{i}$ é o tempo de chegada ao porto; $o_{i}$ é o comprimento total; $f_{i}$ é o calado; $S_{i} \subseteq C$ é o conjunto de cargas transportadas pelo navio; $q_{i c}$ é a quantidade em toneladas da carga $c \in C$ a ser carregada ou descarregada no porto. Cada berço $b \in B$ possui os seguintes parâmetros: $w_{b}$ é comprimento; $h_{b}$ é a profundidade; $R_{b} \subseteq C$ é o conjunto de cargas que podem ser operadas no berço; $l_{b c}$ é a taxa de carregamento/descarregamento da carga $c \in C$.

Define-se, então, que $N^{b} \subseteq V$ como sendo um conjunto de navios que podem ser servidos pelo berço $b \in B$ e, portanto, um navio $i \in V$ só pode ser servido pelo berço $b \in B$ se e somente se $S_{i} \subseteq R_{b}$. Por fim, define-se então que para o problema existe um multigrafo para cada berço $G^{b}=\left(V^{b}, A^{b}\right), \forall b \in B$, onde $V^{b}=N^{b} \cup\{o(b) d(b)\}$ e $A^{b} \subseteq V^{b} \times V^{b}$.

As variáveis de decisão para o modelo matemático proposto são: $x_{i j}^{b}$ que assume valor 1 se o nó $j \in A^{b}$ é atendido depois do nó $i \in A^{b}$ no berço $b \in B$ e zero caso contrário; $T_{i}^{b}$ é o tempo de atracação do navio $i \in V$ no berço $b \in B ; T_{o(b)}^{b}$ e $T_{d(b)}^{b}$ representam respectivamente o início e fim do tempo de operação no berço $b \in B . T_{o(b)}^{b}$ é o tempo em que o primeiro navio atraca no berço $b$ e $T_{d(b)}^{b}$ é o tempo que o último navio deixa o berço $b \in B$.

Após estas definições, são apresentadas a seguir a função objetivo e as restrições do modelo matemático proposto para o PAB-MC. 


\section{Função objetivo}

$\operatorname{Min} v=\sum_{b \in B} \sum_{i \in N^{b}}\left(T_{i}^{b}-a_{i}+\sum_{c \in S_{i}} \frac{q_{i c}}{l_{b c}}\right) \sum_{j \in N^{b} \cup\{d(b)\}} x_{i j}^{b}$

Restrições:

$\sum_{b \in B} \sum_{j \in N^{b} \cup\{d(b)\}} x_{i j}^{b}=1$

$\sum_{j \in N^{b} \cup\{d(b)\}} x_{o(b), j}^{b}=1$

$\sum_{i \in N^{b} \cup\{o(b)\}} x_{i, d(b)}^{b}=1$

$\sum_{j \in N^{b} \cup\{d(b)\}} x_{i j}^{b}=\sum_{j \in N^{b} \cup\{o(b)\}} x_{j i}^{b}$

$\left(T_{i}^{b}+\sum_{c \in S_{i}} \frac{q_{i c}}{l_{b c}}-T_{j}^{b}\right) x_{i j}^{b} \leq 0$

$T_{i}^{b} \geq a_{i} \sum_{j \in N^{b} \cup\{d(b)\}} x_{i j}^{b}$

$\left(w_{b}-o_{i}\right) x_{i j}^{b} \geq 0$

$\left(h_{b}-f_{i}\right) x_{i j}^{b} \geq 0$

$x_{i j}^{b} \in\{0,1\}$ $\forall i \in N^{b}$

$\forall b \in B$

$\forall b \in B$

$\forall b \in B, i \in N^{b}$

$\forall b \in B,(i, j) \in A^{b}$

$\forall b \in B, i \in N^{b}$

$\forall b \in B,(i, j) \in A^{b}$

$\forall b \in B,(i, j) \in A^{b}$

$\forall b \in B,(i, j) \in A^{b}$
A função objetivo (FO), Equação (1), que é não linear, representa o tempo total de permanência de todos os navios no porto e deve ser minimizada. O tempo de permanência é calculado como sendo o tempo de atracação menos o tempo de chegada mais o tempo de operação. O tempo de operação é dado pela tonelagem das cargas do navio dividido pela respectiva taxa de carregamento/descarga do berço.

As Restrições (2) garantem que cada navio é servido uma única vez. Para cada berço $b \in B$, as Restrições (3), (4) e (5) correspondem às restrições de conservação de fluxo de rede de um caminho originado no nó $o(b)$ e terminando no nó $d(b)$. As Restrições (6), que são não lineares, controlam o tempo de atendimento do navio posterior em relação ao navio anterior. As Restrições (7) garantem que o navio só será atendido após a sua chegada ao porto. As Restrições (8) e (9) garantem que um navio pode ser atracado em um berço que tenha comprimento e profundidade maior que o calado do navio. As Restrições (10) garantem que a variável $x_{i j}^{b} \forall b \in B,(i, j) \in A^{b}$ só pode assumir valor 0 ou 1 .

A função objetivo é não linear por causa do termo

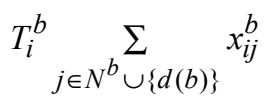

e as Restrições (6) também são não lineares por causa dos termos $T_{i}^{b} x_{i j}^{b}$ e $T_{j}^{b} x_{i j}^{b}$. Para linearizar esses termos, foi utilizado um novo parâmetro $H p$ que representa o horizonte de planejamento. Também foi definida uma nova variável $z_{i}^{b}$ que representa o tempo de atracação de um navio $i \in V$ no berço $b \in B$. Para completar a linearização do modelo, quatro restrições (11)-(14) são propostas. 
O modelo proposto pode ser linearizado agregando-se o parâmetro $H p$, a variável $z_{i}^{b}$ e as Restrições (11) a (14). Além disso, a FO, Equação (1) deve ser substi- tuída pela Equação (15) e as Restrições (6) devem ser substituídas pelas Restrições (16).

$$
\begin{aligned}
& z_{i}^{b} \leq H p \sum_{j \in N^{b} \cup\{d(b)\}} x_{i j}^{b} \\
& \forall b \in B, i \in A^{b} \\
& z_{i}^{b} \leq T_{i}^{b} \\
& \forall b \in B, i \in A^{b} \\
& z_{i}^{b} \geq T_{i}^{b}-H p\left(1-\sum_{j \in N^{b} \cup\{d(b)\}} x_{i j}^{b}\right) \\
& \forall b \in B, i \in A^{b} \\
& z_{i}^{b} \geq 0 \\
& \forall b \in B, i \in A^{b} \\
& \operatorname{MIN} \quad v=\sum_{b \in B} \sum_{i \in N^{b}}\left(T_{i}^{b}-\left(\left(a_{i}-\sum_{c \in S_{i}} \frac{q_{i c}}{l_{b c}}\right) \sum_{j \in N^{b} \cup\{d(b)\}} x_{i j}^{b}\right)\right) \\
& T_{i}^{b}+\left(\sum_{c \in S_{i}} \frac{q_{i c}}{l_{b c}} x_{i j}^{b}\right)-T_{j}^{b} \leq 0 \\
& \forall b \in B,(i, j) \in A^{b}
\end{aligned}
$$

\section{META-HEURÍSTICA SIMULATED ANNEALING PROPOSTA}

Sabendo-se que o PRV é considerado NP-Hard e, por conseguinte, o PRV-MD-JT também é NP-Hard, infere-se que o modelo proposto neste artigo também seja NP-Hard. Assim, problemas NP-Hard são problemas difíceis de encontrar soluções ótimas devido à sua complexidade combinatória, sobretudo, para instâncias maiores e, então, este artigo propõe uma meta-heurística Simulated Annealing para resolução do modelo matemático proposto, o PAB-MC.

O SA funciona como um método de busca local que aceita soluções piores para escapar de ótimos locais. O SA começa sua busca a partir de uma solução inicial qualquer. $\mathrm{O}$ procedimento principal consiste em um laço que gera aleatoriamente, em cada iteração, um único vizinho $S$ ' da solução corrente $\mathrm{S}$ por meio de movimentos aleatórios de troca. Uma nova solução com valor da função objetivo pior do que a solução atual pode ser aceita como a nova solução corrente, com uma probabilidade determinada pelos critérios de Metropolis (KIRKPATRICK et al., 1983). A ideia é não restringir os movimentos do algoritmo de busca nas direções que melhoram o valor da função objetivo, permitindo movimentos que deterioram o valor da função objetivo com base em probabilidade que é inversa à temperatura que o SA está no momento do teste (TING et al., 2013).

Para a implementação da meta-heurística, o código programado em linguagem $\mathrm{C}$ realiza a leitura dos dados referentes aos navios que solicitam atracação aos berços do porto. Assim, os dados de entrada para cada navio são o tempo de chegada, o comprimento, o calado e a quantidade de cada tipo de carga que está sendo transportada e deve ser carregada e/ou descarregada. Para cada berço, os dados de entrada correspondem ao comprimento, à profundidade e a taxa de carregamento e/ou descarregamento para cada tipo de carga. Além da entrada de dados, o código executa três sub-rotinas principais: 1) Pré-processamento; 2) Inicialização; e 3) Simulated Annealing.

Uma particularidade da meta-heurística proposta é a utilização da sub-rotina de Pré-processamento que foi concebida devido as particularidades do problema estudado com a função de auxiliar na escolha de navios para os movimentos de troca. Isto é, como o problema possui restrição de tipos de cargas que podem ser operadas em cada berço além da restrição do calado do navio em relação à profundidade do berço, seria dispendido um tempo computacional considerável somente para encontrar um berço viável para os movimentos de troca. Portanto, como estratégia para contornar essa característica do problema tratado, foi utilizada uma matriz de atracação $M_{b x}$. Essa matriz possui $b$ linhas representando cada um dos berços, e nas colunas os respectivos navios que podem atracar no berço $b$. Isto é, a matriz representa quais navios são passíveis de atracação em cada um dos berços. Essa informação a priori permite que a escolha aleatória do navio, após a escolha do berço, seja feita somente dentro do conjunto de navios que possam atracar neste berço. Esta matriz é gerada na sub-rotina Pré-processamento que tem seu pseudocódigo apresentado na Figura 3. 
PARA (cada berço $b \in B$ ) FAÇA

$\mathrm{x}=1$;

PARA (cada navio $i \in V$ ) FAÇA

atracar $=1$;

SE (comprimento do navio $i>$ comprimento do berço $b$ ) OU

(calado do navio $i>$ profundidade do berço $b$ ) ENTÃO

atracar $=0$;

SENÃO

Para (cada carga $c \in C$ ) FAÇA

SE (a quantidade da carga $c$ transportada pelo navio $i>0$ ) E

(taxa de carregamento/descarga do berço $b$ para a carga $c=0$ ) ENTÃO atracar $=0$;

FIM-SE

FIM-PARA

FIM-SE

SE (atracar $=1)$ ENTÃO

$M_{b x}=i$;

$\mathrm{x}=\mathrm{x}+1$;

FIM-SE

FIM-PARA

FIM-PARA

Figura 3: Pseudocódigo da sub-rotina Pré-processamento

A sub-rotina Inicialização é responsável por gerar a solução inicial $S$ que será utilizada no $S A$. O algoritmo consiste em alocar os navios, um a um, cada qual em um berço que possa atendê-lo, dadas as restrições do problema, e que possua o menor número de navios agendados no momento; veja a Figura 4.

PARA (cada navio $i \in V$ ) FAÇA

SELECIONAR ((berço $b \in B$ com menor número de navios atendidos) E

(comprimento do navio $i<$ comprimento do berço $b$ ) $\mathrm{E}$

(calado do navio $i<$ profundidade do berço $b$ ))

DESIGNAR (navio $i$ ao berço $b$ );

FIM-PARA

Figura 4: Pseudocódigo da sub-rotina Inicialização

A sub-rotina Simulated Annealing é responsável por definir em qual berço o navio irá atracar, sua posição na fila de atracação do berço e o tempo de atracação. A implementação é baseada em Mauri et al. (2008a) e foram realizas as seguintes adaptações: 1) adição do parâmetro tempoMaxSA; 2) exclusão dos comandos de gerar e avaliar a solução inicial $S$, visto que a sub-rotina Inicialização realiza essas funções; 3) a meta-heurística é executada enquanto o tempo de execução for inferior ao parâmetro tempoMaxSA; 4) são realizados tantos reaquecimentos quanto possíveis ao longo do tempo de exe- cução; 5) não é realizado após os movimentos de troca o reordenamento pelo tempo de chegada dos navios em cada berço alterado; e 6) no reaquecimento não se eleva a temperatura à temperatura inicial, utiliza-se a fórmula proposta por Júnior et al. (2005) para reduzir a temperatura, levando assim, a cada reaquecimento a diminuição da probabilidade de se aceitar uma solução pior do que a solução atual, tendo em vista que ao fim de um ciclo de temperatura, a meta-heurística já esta convergindo para uma solução. O pseudocódigo da sub-rotina Simulated Annealing é apresentado na Figura 5. 


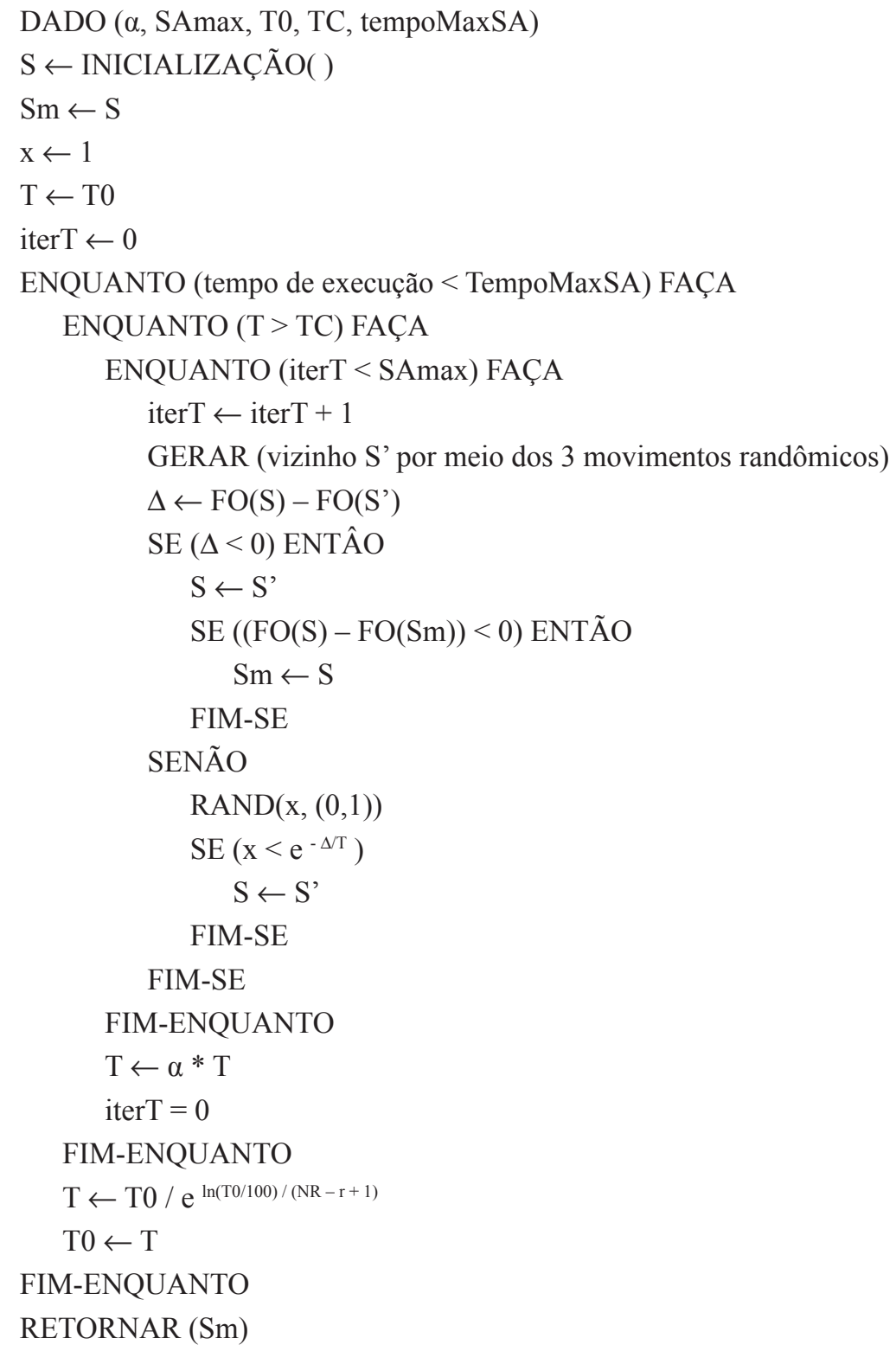

Figura 5: Pseudocódigo da sub-rotina Simulated Annealing

A função GERAR no pseudocódigo da sub-rotina $\mathrm{Si}$ mulated Annealing, Figura 4, gera vizinhos a partir da solução S. Para a geração dos vizinhos são utilizados três movimentos de troca: 1) Reordenar navios, 2) Realocar navio e 3) Trocar navios. O parâmetro $\alpha$ representa a taxa de resfriamento adotada, o parâmetro SAMax representa o número de iterações para cada temperatura pesquisada, TC é a temperatura de congelamento ou de parada do SA e tempoMaxSA representa o tempo máximo de execução do SA.

O movimento Reordenar navios, Figura 6, consiste em selecionar um berço qualquer aleatoriamente pertencente à solução, B1, selecionar um navio qualquer aleatoriamente atendido por esse berço, N2, selecionar outro navio no mesmo berço diferente do primeiro também de forma aleatória, N4, e trocar os dois navios de posição de atendimento. Após a troca, devem-se ajustar os tempos de atendimento dos navios que vão atracar no berço, pois em função do tempo de operação dos dois navios que foram trocados podem ocorrer mudanças no tempo de atracação dos outros navios.
O movimento Trocar navios, Figura 7 (a), consiste em selecionar aleatoriamente dois berços quaisquer diferentes um do outro, B1 e B2, selecionar um navio qualquer em cada um dos dois berços, $\mathrm{N} 2$ e $\mathrm{N} 9$, respectivamente, respeitando o fato que o navio $\mathrm{N} 2$ possa ser operado no berço B2 e o navio N9 possa ser operado no berço B1, caso contrário, um novo navio deverá ser escolhido também de forma aleatória. Após esta verificação, os dois navios selecionados são trocados de berço ajustando os tempos de atendimento dos navios atracados em ambos os berços, pois em função do tempo de operação dos dois navios que foram trocados podem ocorrer mudanças no tempo de atracação dos outros navios posteriores à posição de atracação dos navios trocados.

O movimento Realocar navio, Figura 7 (b), consiste em selecionar de forma aleatória um berço qualquer da solução, B1, e para este berço selecionar de forma aleatória um navio qualquer, N2. Selecionar outro berço também de forma aleatória diferente do primeiro berço selecionado, no 

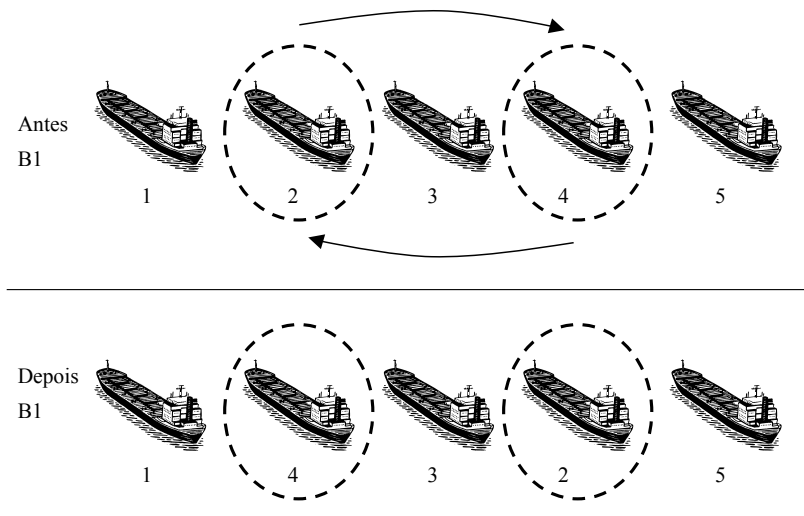

Figura 6: Movimento Reordenar navios

caso, B2, que pode operar o navio N2, caso contrário, outro berço deverá ser selecionado. Após estas escolhas, deve-se retirar o navio escolhido, N2, do berço B1 e inseri-lo no final da lista de navios atendidos do berço B2. Os navios do berço B1 posteriores ao navio retirado N2 deverão ter seus tempos de atracação recalculados.

A partir da estrutura de vizinhança explicada anteriormente, a função GERAR foi implementada de modo que cada solução vizinha é gerada por apenas um desses movimentos. A decisão de qual movimento deve ser realizado é tomada de forma aleatória, porém uniformemente distribuída, possibilitando assim uma boa diversidade entre as soluções intermediárias geradas, e consequentemente uma boa exploração do espaço de soluções.

\section{APRESENTAÇÃO E ANÁLISE DOS RESULTADOS}

O modelo matemático foi executado no solver CPLEX 12.6 em um computador com processador Intel i7 e
16GB de memória RAM, com o tempo limite de execução definido em 7.200 segundos, enquanto que a meta-heurística foi programada em linguagem $\mathrm{C}$ e executada no mesmo computador. Após vários testes com diferentes valores, pôde-se constatar que os parâmetros utilizados no SA que deram melhor resultado foram: $\alpha=0,975$, SAmax $=1000$, $\mathrm{T} 0=20000$ e $\mathrm{TC}=0,01 \mathrm{e}$, portanto, foram os valores usados na execução do SA.

Foram criadas inicialmente cinco instâncias com 10 navios para validação do SA. Definindo-se a quantidade de berços em 4, 7, 9, 11 e 15. As instâncias de testes foram criadas com esses tamanhos para obter a solução ótima com o CPLEX e, então, foi possível usá-las como validação da solução do SA.

Depois, foram elaboradas mais três instâncias com base nos dados reais do Complexo Portuário de Tubarão. Cada uma delas tinha os sete berços operados pela Vale $\mathrm{S} / \mathrm{A}$, definindo-se a quantidade de navios em 100, 150 e 250. Finalmente, foram geradas mais três instâncias que refletem o cenário de possível expansão das operações, na qual a empresa pode passar a operar outros quatro berços. Este cenário é composto de 11 berços, e 100, 150 e 250 navios em cada instância.

Tendo em vista ser possível encontrar soluções diferentes em cada execução do SA para uma mesma instância devido à natureza aleatória do processo do SA para obter a solução, o SA foi executado 10 vezes para cada instância e foi apurado após as 10 execuções o desvio médio como medida de dispersão.

Os resultados estão apresentados na Tabela 1 , na qual a coluna Instância representa o nome da instância, sendo que sua estrutura é formada por $\mathrm{B} b b \mathrm{~N} n n n$, onde $b b$ é a quantidade de berços e nnn é a quantidade de navios da instância. As cinco colunas subsequentes representam os resultados alcançados pelo CPLEX, sendo a coluna FO o valor da solução ótima alcançada pelo CPLEX. A col-
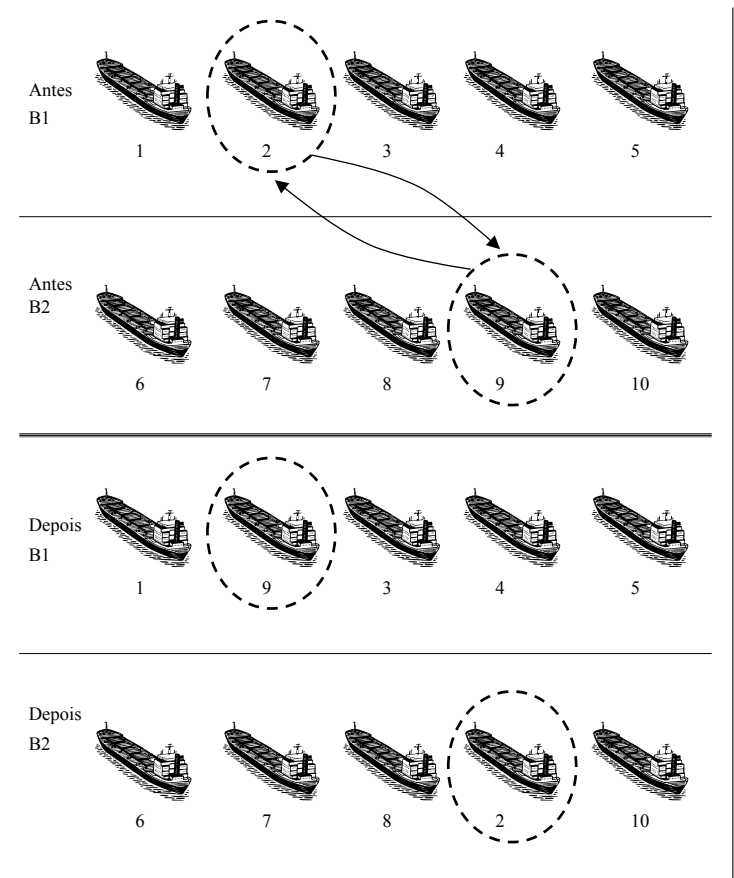

( a )
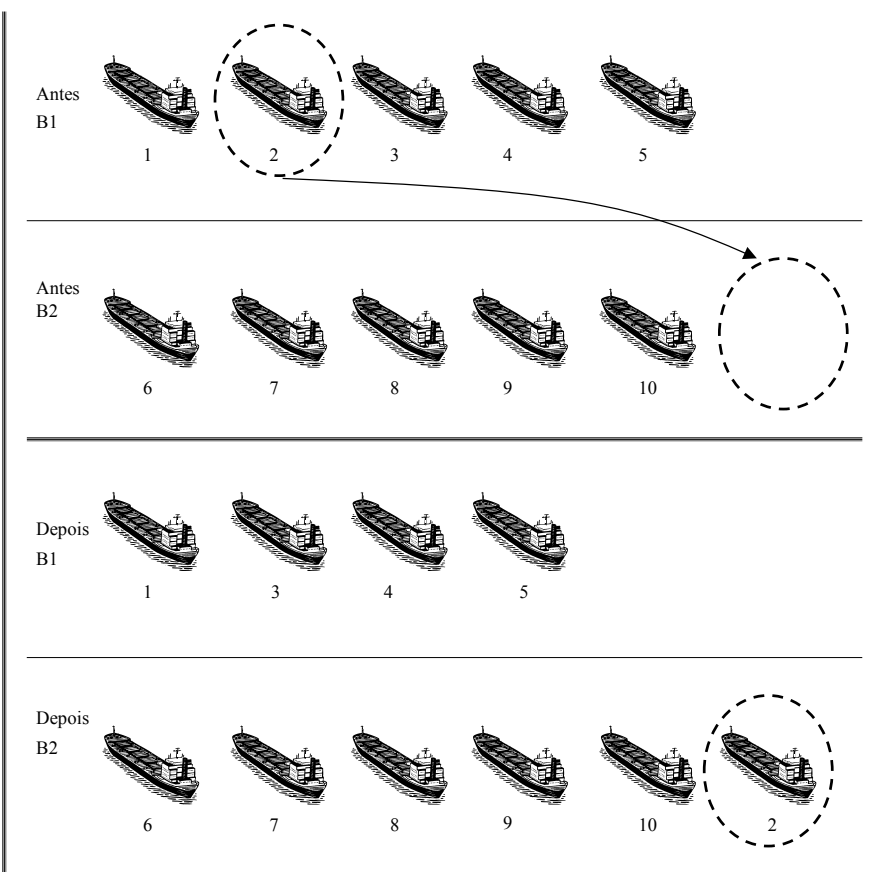

(b)

Figura 7: (a) Movimento Trocar navios; (b) Movimento Realocar navios 
Tabela 1: Resultados do CPLEX e do SA

\begin{tabular}{|c|c|c|c|c|c|c|c|c|c|}
\hline \multirow[b]{2}{*}{ Instância } & \multicolumn{5}{|c|}{ CPLEX } & \multicolumn{4}{|c|}{$S A$} \\
\hline & $\begin{array}{c}\text { FO } \\
\left(\mathrm{h} \times 10^{3}\right)\end{array}$ & $\begin{array}{l}\text { Upper } \\
\text { Bound } \\
\left(\mathrm{h} \times 10^{3}\right)\end{array}$ & $\begin{array}{l}\text { Lower } \\
\text { Bound } \\
\left(\mathrm{h} \times 10^{3}\right)\end{array}$ & $\begin{array}{l}\text { Gap } \\
(\%)\end{array}$ & $\begin{array}{l}\text { Tempo } \\
\text { Execu- } \\
\text { ção (s) }\end{array}$ & $\begin{array}{c}\text { Melhor } \\
\text { FO } \\
\left(\mathrm{h} \times 10^{3}\right)\end{array}$ & $\begin{array}{c}\text { FO } \\
\text { Média } \\
\left(\mathrm{h} \times 10^{3}\right)\end{array}$ & $\begin{array}{c}\text { Desvio } \\
\text { Médio } \\
(\%)\end{array}$ & $\begin{array}{l}\text { Tempo } \\
\text { Execu- } \\
\text { ção (s) }\end{array}$ \\
\hline B04N010 & 517,69 & - & - & 0,0 & 2,29 & 517,69 & 517,69 & 0,0 & 120,0 \\
\hline B07N010 & 555,98 & - & - & 0,0 & 0,89 & 555,98 & 555,98 & 0,0 & 120,0 \\
\hline B09N010 & 661,40 & - & - & 0,0 & 4,28 & 661,40 & 661,40 & 0,0 & 120,0 \\
\hline B11N010 & 241,02 & - & - & 0,0 & 2,77 & 241,02 & 241,02 & 0,0 & 120,0 \\
\hline $\mathrm{B} 15 \mathrm{~N} 010$ & 115,82 & - & - & 0,0 & 0,59 & 115,82 & 115,82 & 0,0 & 120,0 \\
\hline $\mathrm{B} 07 \mathrm{~N} 100$ & - & 27,87 & 3,39 & 87,86 & 7200,0 & 24,48 & 26,14 & 2,27 & 300,0 \\
\hline $\mathrm{B} 07 \mathrm{~N} 150$ & - & 67,64 & 6,83 & 89,90 & 7200,0 & 51,69 & 52,55 & 0,72 & 300,0 \\
\hline $\mathrm{B} 07 \mathrm{~N} 250$ & - & 254,72 & 7,32 & 97,13 & 7200,0 & 128,57 & 131,97 & 1,12 & 300,0 \\
\hline B11N100 & - & 6,79 & 1,45 & 78,69 & 7200,0 & 5,06 & 5,28 & 2,79 & 300,0 \\
\hline $\mathrm{B} 11 \mathrm{~N} 150$ & - & 53,74 & 2,17 & 95,96 & 7200,0 & 12,15 & 12,80 & 3,06 & 300,0 \\
\hline $\mathrm{B} 11 \mathrm{~N} 250$ & - & 287,97 & 3,74 & 98,70 & 7200,0 & 30,23 & 32,21 & 3,79 & 300,0 \\
\hline
\end{tabular}

una Upper Bound (UB) e a coluna Lower Bound (LB), que representam respectivamente o upper bound e lower bound, respectivamente, encontrados pelo CPLEX após 7.200,0 segundos de execução. A coluna GAP é calculada pela fórmula $G A P=100 \times(U B-L B) / U B$. A coluna Tempo de Execução representa o tempo que o CPLEX levou para chegar ao resultado. As quatro colunas subsequentes representam o resultado alcançado pelo SA sendo que a coluna Melhor FO representa a melhor solução encontrada pelo SA, a coluna FO Média representa a média das soluções encontradas nas 10 execuções do SA para a instância, a coluna Desvio Médio representa o desvio médio e a coluna Tempo de Execução é o tempo que o SA foi executado.

Para as instâncias B04N010, B07N010, B09N010, B11N010 e B15N010, em função do pequeno número de navios, o CPLEX foi capaz de encontrar uma solução ótima para o modelo matemático em um pequeno tempo de execução. Ao comparar os resultados obtidos pelo CPLEX e os resultados obtidos pelo SA, pode-se perceber que o SA foi capaz de encontrar uma solução igual à solução ótima encontrada pelo CPLEX para todas as instâncias de teste. Portanto, é possível que o SA seja capaz de convergir para a solução ótima nas demais instâncias ou então encontrar uma solução próxima da ótima dado um tempo de execução suficiente para tal. Vale ressaltar que a coluna Desvio Médio foi igual a 0,0\% para todas as instâncias, ou seja, em todas as 10 execuções do SA, as soluções encontradas foram iguais.

Os resultados do SA para as instâncias B07N100, B07N150, B07N250, B11N100, B11N150 e B11N250 mostraram que o CPLEX não foi capaz de achar a solução ótima dentro do tempo de execução de duas horas apresentando valores de GAP muito altos. Assim, foram utilizados os valores de UB e de LB alcançados pelo CPLEX como alternativa para comparação dos resultados. Esses valores representam, na prática, um limite superior e in- ferior, respectivamente, para a solução encontrada pelo SA. É possível perceber pelos valores do GAP da Tabela 1 que os limites apresentados pelo modelo matemático estão longe de convergirem para a otimalidade. Todavia, as soluções apresentadas pelo SA estão dentro dos limites encontrados pelo CPLEX, UB e LB, implicando em uma solução viável.

Assim, pode-se mostrar que o SA proposto foi capaz de resolver os problemas reais apresentados no Complexo Portuário de Tubarão, inclusive considerando as futuras expansões do porto. Com base nas dimensões das instâncias, pode-se dizer que o SA proposto pode ser utilizado em qualquer porto do Brasil e do mundo, tornando-se uma ferramenta útil para o planejamento das operações portuárias.

\section{CONCLUSÕES}

Este artigo propôs um novo modelo matemático para o Problema de Alocação de Berços para Múltiplas Cargas e, propôs, ainda, uma meta-heurística SA para o mesmo problema. O modelo e o SA foram aplicados a um porto de granel, e vale ressaltar que até o momento poucos estudos do PAB foram publicados sobre portos a granel. O problema tratado tem uma característica que o difere dos demais por considerar que o tempo de operação não é conhecido a priori e é dependente da carga e do berço que o navio irá atracar.

Visto que os modelos matemáticos propostos para resolver o PAB não são capazes de apresentar soluções ótimas para instâncias de maior tamanho, a utilização de meta-heurísticas é tida como um caminho natural para se conseguir soluções próximas da ótima em tempos de execução comparativamente baixos. Os resultados mostram o potencial da abordagem apresentada, na qual soluções de boa qualidade são obtidas para instâncias relativamente grandes e em tempos de execução comparativamente baixos. Tais soluções propiciam reduções de custos, uma vez que há diminuição do tempo em que os navios permanecem no porto 
e, sobremaneira, há a possibilidade de geração de resultados financeiros positivos para a empresa.

Espera-se que este trabalho tenha contribuído para evidenciar a importância de um agendamento eficaz da atracação de navios em portos, e que o escasso estudo do PAB-MC possa ser considerado como um ponto motivador de trabalhos futuros. O PAB-MC foi aplicado a um porto granel, porém, ele representa a realidade de muitos portos de carga geral também. Por fim, sugere-se como continuidade deste trabalho a aplicação do PAB-MC a outros portos de granel, bem como portos de carga geral.

\section{AGRADECIMENTOS}

Os autores agradecem à FAPES (458/2013 e 67627153/2014), ao CNPq (477357/2013-0, 313408/20149 e 303052/2013-9 e 454569/2014-9) e à CAPES pelo apoio financeiro e a Vale S/A pelo apoio ao fornecimento dos dados do problema.

\section{REFERÊNCIAS}

ANTAQ. (2013) Boletim anual de movimentação de cargas. 2013. Disponível em: <http://www.antaq.gov.br/Portal/Anuarios/ Anuario2013/Tabelas/AnaliseMovimentacaoPortuaria.pdf>. Acesso em: junho de 2016.

Barros, V. H.; Costa, T. S.; Oliveira, A. C. M.; Lorena, L. A. N. (2011) Model and heuristic for berth allocation in tidal bulk ports with stock level constraints. Computers and Industrial Engineering, v. 60, n. 4, p. 606-613. DOI: 10.1016/j.cie.2010.12.018

Bierwirth, C.; Meisel, F. (2010) A survey of berth allocation and quay crane scheduling problems in container terminals. European Journal of Operational Research, v. 202, n. 3, p. 615-627. DOI: 10.1016/j.ejor.2009.05.031

Buhrkal, K.; Zuglian, S.; Ropke, S.; Larsen, J.; Lusby, R. (2011) Models for the discrete berth allocation problem: A computational comparison. Transportation Research Part E, v. 47, p. 461473. DOI: $10.1016 /$ j.tre.2010.11.016

Cordeau, J. F.; Laporte, G.; Legato, P.; Moccia, L. (2005) Models and tabu search heuristics for the berth-allocation problem. Transportation Science, v.39, n.4, p. 526-538. DOI: 10.1287/ trsc. 1050.0120

Golias, M. M. (2007) The discrete and continuous berth allocation problem: Models and algorithms. Dissertação (Doctor of Philosophy Graduate Program in Civil and Environmental Engineering). The State University of New Jersey, New Brunswick.

Guan, Y.; Xiao, W. Q.; Cheung, R. K.; Li, C. L. (2002) A multiprocessor task scheduling model for berth allocation: heuristic and worst case analysis. Operations Research Letters, v. 30, n. 5, p. 343-350. DOI: 10.1016/S0167-6377(02)00147-5

Hansen, P., Oguz, C. (2003) A note on formulations of static and dynamic berth allocation problems. Les Cahiers du GERAD 30, $1-17$.
Hansen, P.; Oguz, C.; Mladenovic, N. (2008) Variable neighborhood search for minimum cost berth allocation. European Journal of Operational Research, v. 191, n. 3, p. 636-649. DOI: 10.1016/j.ejor.2006.12.057

Imai, A.; Nagaiwa, K.; Tat, C. W. (1997) Efficient planning of berth allocation for container terminals in Asia. Journal of Advanced Transportation, v. 31, n. 1, p. 75-94, 1997. DOI: 10.1002/atr.5670310107

Imai, A., Nishimura, E., Papadimitriou, S. (2003) Berth allocation with service priority. Transportation Research Part B, v. 37, n. 5, p. 437-457. DOI: 10.1016/S01912615(02)00023-1

Imai, A.; Sun, X.; Nishimura, E.; Papadimitriou, S. (2005) Berth allocation in a container port: using a continuous location space approach. Transportation Research Part B, v. 39, n. 3, p. 199-221. DOI: $10.1016 /$ j.trb.2004.04.004

Imai, A., Nishimura, E., Papadimitriou, S. (2008) Berthing ships at a multi-user container terminal with a limited quay capacity. Transportation Research Part E, v. 44, n. 1, p. 136151. DOI: $10.1016 /$ j.tre.2006.05.002

Imai, A.; Chen, H. C.; Nishimura, E.; Papadimitriou, S. (2008) The simultaneous berth and quay crane allocation problem. Transportation Research Part E, v. 44, n. 5, p. 900-920. DOI: 10.1016/j.tre.2007.03.003

Imai, A.; Nishimura, E.; Papadimitriou, S. (2001) The dynamic berth allocation problem for a container port. Transportation Research Part B, v. 35, n. 4, p. 401-417. DOI: 10.1016/S01912615(99)00057-0

Júnior, A. D. C. G., Souza, M. J. F., Martins, A. X. (2005). Simulated annealing aplicado à resolução do problema de roteamento de veículos com janela de tempo. TRANSPORTES, v. 13, n. 2, p. 5-20. DOI: $10.14295 /$ transportes.v13i2.98

Kim, K. H.; Moon, K.c. (2003) Berth scheduling by simulated annealing. Transportation Research Part B, v. 37, n. 6, p. 541560. DOI: 10.1016/S0191-2615(02)00027-9

Kirkpatrick, S.; Gelatt Jr, C. D.; Vecchi, M. P. (1983) Optimization by Simulated Annealing. Science, v. 220, n. 4598, p. 671680. DOI: $10.1126 /$ science.220.4598.671

Lai, K. K.; Shih, K. (1992) A study of container berth allocation. Journal of Advanced Transportation, v. 26, n. 1, p. 45-60. DOI: $10.1002 /$ atr.5670260105

Li, C-L.; Cai, X.; Lee, C-Y. (1998) Scheduling with multiplejob-on-one-processor pattern. IIE Transactions, v. 30, n. 5, p. 433-445. DOI: 10.1023/A:1026487022192

Lim, A. (1998) The berth planning problem. Operations Research Letters, v. 22, n. 2-3, p. 105-110. DOI: 10.1016/S01676377(98)00010-8 
Mauri, G. R.; Oliveira, A. C. M.; Lorena, L. A. N. (2008a) Heurística baseada no Simulated Annealing aplicada ao problema de alocação de berços. GEPROS - Gestão da Produção, Operações e Sistemas, Ano 3, v. 1, n. 1, p. 113-127.

Mauri, G. R.; Oliveira, A. C. M.; Lorena, L. A. N. (2008b) A hybrid column generation approach for the berth allocation problem. In: VAN EMERT, J.; COTTA, C. (Eds.), EvoCOP 2008, Lecture Notes in Computer Science, v. 4972, p. 110-122, Springer. DOI: 10.1007/978-3-540-78604-7_10

Mauri, G.r., Oliveira, A.c.m., Lorena, L.a.n. (2010) Resolução do Problema de Alocação de Berços Através de Uma Técnica de Geração de Colunas. Pesquisa Operacional, v. 30, n. 3, p. 547-562. DOI: 10.1590/S0101-74382010000300003

Monaco, M. F.; Sammarra, M. (2007) The berth allocation problem: A Strong formulation solved by a lagrangean approach. Transportation Science, v. 41, n. 2, p. 265-280. DOI: 10.1287/ trsc. 1060.0171

Nishimura, E.; Imai, A.; Papadimitriou, S. (2001) Berth allocation planning in the public berth system by genetic algorithms. European Journal of Operational Research, v. 131, n. 2, p. 282292. DOI: $10.1016 / \mathrm{S} 0377-2217(00) 00128-4$

Oliveira, R. M.; Mauri, G. R.; Lorena, L. A. N. (2012) Clustering search heuristics for solving a continuous berth allocation problem. Lecture Notes in Computer Science, v. 7245, p. 49-62. DOI: $10.1007 / 978-3-642-29124-1 \_5$

Park, K. T.; Kim, K. H. (2002) Berth scheduling for container terminals by using a sub-gradient optimization technique. Journal of the Operational Research Society, v. 53, p. 1054-1062. DOI: 10.1057/palgrave.jors.2601412

Rodrigues, M. H. P.; Machado, C. M. S.; Lima, M. L. P. (2013) Simulated annealing aplicado ao problema de alocação de berços. Journal of Transport Literature, v. 7, n. 3, p. 117-136. DOI: 10.1590/S2238-10312013000300006

Rodrigues, P. H.; Rosa, R. A.; Arpini, B. P.; Bissoli, A. L.; Ribeiro, G. M. (2015) Planejamento de atracação de navios por meio de um modelo matemático para o problema de alocação de berços contínuos com descontinuidade do cais. TRANSPORTES, v. 23 , n. 1 , p. 37-46. DOI: $10.4237 /$ transportes.v23i1.681

Rosa, R. A.; Resendo, L. C.; Lopes, F. T. (2013) Proposta de um Modelo Matemático para o Problema de Alocação de Berços para Múltiplas Cargas (PAB-MC) com Restrições Temporais e Espaciais. In: CNT Confederação Nacional do Transporte. (Org.). Transporte em Transformação XVII. 1ed. Brasília: Positiva, v. 1, p. 133-150.

Sindamares. (2013) Administração do Complexo Portuário de Tubarão e Praia Mole (TPM). Disponível em: <http://www. sindamares.com.br/tubarao.html>. Acesso em: Julho de 2013.

Teixeira, R. B.; Cunha, C. B. (2012) Modelo integrado para seleção de cargas e reposicionamento de contêineres vazios no transporte marítimo. TRANSPORTES, v. 20, n. 1, p. 59-70. DOI: $10.4237 /$ transportes.v20i1.442
Theofanis, S., Boile, M., Golias, M. (2007) An optimization based genetic algorithm heuristic for the berth allocation problem. IEEE Congress on Evolutionary Computation 2007 (CEC 2007). IEEE Computer Society, Washington DC, pp. 4439-4445. DOI: 10.1109/CEC.2007.4425052

Ting, C. J.; Lin, S. W.; Wu, K. C. (2013) The Continuous Berth Allocation Problem by Simulated Annealing, In: APIEMS Conference 2013 Proceedings, Cebu, Philippines.

Tong, C. J.; Lau, H. C.; Lim, A. (1999) Ant colony optimization for the ship berthing problem. In: THIAGARAJAN, P.S.; Yap, R. (Eds.), Fifth Asian Computing Science Conference (ASIAN'99), Lecture Notes in Computer Science, v.1742, p. 359-370, Springer. DOI: 10.1007/3-540-46674-6_30

Vervloet, H. L., Rosa, R. A. (2016) Modelo matemático para planejamento da atracação de navios sob o contrato de charter party. TRANSPORTES, no prelo. 